\title{
A Cryogenic Process for Antisolvent-Free High-Performance Perovskite Solar Cells
}

\author{
Annie Ng, Zhiwei Ren, Hanlin Hu, Patrick W. K. Fong, Qian Shen, Sin Hang Cheung, \\ Pingli Qin, Jin-Wook Lee, Aleksandra B. Djurišić, Shu Kong So, Gang Li, Yang Yang, \\ and Charles Surya*
}

\begin{abstract}
A cryogenic process is introduced to control the crystallization of perovskite layers, eliminating the need for the use of environmentally harmful antisolvents. This process enables decoupling of the nucleation and the crystallization phases by inhibiting chemical reactions in as-cast precursor films rapidly cooled down by immersion in liquid nitrogen. The cooling is followed by blow-drying with nitrogen gas, which induces uniform precipitation of precursors due to the supersaturation of precursors in the residual solvents at very low temperature, while at the same time enhancing the evaporation of the residual solvents and preventing the ordered precursors/perovskite from redissolving into the residual solvents. Using the proposed techniques, the crystallization process can be initiated after the formation of a uniform precursor seed layer. The process is generally applicable to improve the performance of solar cells using perovskite films with different compositions, as demonstrated on three different types of mixed halide perovskites. A champion power conversion efficiency (PCE) of $21.4 \%$ with open-circuit voltage $\left(V_{O C}\right)=1.14 \mathrm{~V}$, short-circuit current density $\left(J_{s C}\right)=23.5 \mathrm{~mA} \mathrm{~cm}^{-2}$, and fill factor $(\mathrm{FF})=0.80$ is achieved using the proposed cryogenic process.
\end{abstract}

When humanity in the modern world consumes ever-increasing energy for the development of the infrastructure, growth of the economy and to raise the standard of living, securing clean and sustainable energy resources becomes a critical issue of concern. Among different renewable energy resources, solar energy, which is abundant in nature, is one of the most promising candidates to address the global energy challenge. However, widespread use of solar power requires the development of cost-effective high-performance solar cells. Recently, the emergence of the hybrid organometal halide perovskite as photovoltaic absorbers had led to a breakthrough in the field of solar technologies. The perovskite-based solar cells (PSCs) had accomplished a dramatic enhancement in the power conversion efficiency (PCE) from $3.8 \%$ in $2009^{[1]}$ to a lately announced certificated PCE of $23.3 \%$, ${ }^{[2]}$ outperforming not only other emerging technologies, but also a number of well-established photovoltaic technologies. The superior properties of hybrid organometal halide perovskite materials include high absorption coefficients, tunable bandgaps, long carrier diffusion length, high carrier mobility, and low exciton binding energy making the material highly suitable for photovoltaic applications. ${ }^{\left[{ }^{3-6]}\right.}$ The theoretical maximum PCE for the $\mathrm{CH}_{3} \mathrm{NH}_{3} \mathrm{PbI}_{3-x} \mathrm{Cl}_{x}$-based PSCs is found to be $31.4 \%,{ }^{[7]}$ which approaches the Shockley-Queisser limit of 33\% achieved by gallium arsenide solar cells..$^{[8]}$

Tremendous research efforts had been devoted for optimizing the device architecture, as well as deposition techniques and composition of different layers, in particular the perovskite absorber. ${ }^{[9-15]}$ It is well known that high efficiency and good stability PSCs require certain desirable perovskite film properties such as low defect density, high crystallinity, good coverage, and uniformity. ${ }^{[16-19]}$ The perovskite films can be prepared by different methods, such as solution techniques, ${ }^{[20,21]}$ thermal evaporation, ${ }^{[22-24]}$ and a combination of vapor and the solution
Dr. A. Ng

Department of Electrical and Computer Engineering

School of Engineering

Nazarbayev University

Astana 010000, Kazakhstan

Dr. Z. Ren, Dr. H. Hu, Dr. P. W. K. Fong, Q. Shen, Dr. P. Qin, Prof. G. Li

Department of Electronic and Information Engineering

The Hong Kong Polytechnic University

Hong Kong S.A.R.

S. H. Cheung, Prof. S. K. So

Department of Physics

Hong Kong Baptist University

Hong Kong S.A.R.

\author{
Dr. J.-W. Lee, Prof. Y. Yang \\ Department of Materials Science and Engineering \\ University of California \\ Los Angeles, CA 90095-9000, USA \\ Prof. A. B. Djurišić \\ Department of Physics \\ The University of Hong Kong \\ Pokfulam, Hong Kong S.A.R. \\ Prof. C. Surya \\ School of Engineering \\ Nazarbayev University \\ Astana, Kazakhstan \\ E-mail: charles.surya@nu.edu.kz
}

DOI: $10.1002 / \mathrm{adma} .201804402$ 
processes. ${ }^{[25-27]}$ The majority of the early works reported focused on simple 1 -step or 2 -step solution techniques. ${ }^{[1,3,20]}$ Difficulties in achieving good coverage and uniformity of perovskite films on the substrates in large scale are the main concerns of using these solution-based processes. To address these issues, solvent engineering and the use of antisolvent dripping were introduced. ${ }^{28-30]}$ Solvent engineering approach relies on the use of a mixture of solvents, such as dimethylformamide $(\mathrm{DMF})^{[28]}$ or $\gamma$-butyrolactone, ${ }^{[30]}$ with dimethylsulphoxide (DMSO), for the 1-step spin-coating technique. This enables more controlled reaction between the perovskite precursors due to the formation of a $\mathrm{CH}_{3} \mathrm{NH}_{3} \mathrm{I}-\mathrm{PbI}_{2}$-DMSO intermediary phase. ${ }^{[30]}$ The main function of the DMSO was to retard the rapid reaction between $\mathrm{PbI}_{2}$ and $\mathrm{CH}_{3} \mathrm{NH}_{3} \mathrm{I}$ during solvent evaporation in the spinning step due to the interaction among Lewis base DMSO, iodide and Lewis acid $\mathrm{PbI}_{2}$. At the final stage, the residual DMSO was removed by thermal annealing, resulting in perovskite films with high crystallinity and uniform grain distribution. The introduction of antisolvent assisted crystallization during spin-coating further improves the quality of the perovskite layer by promoting rapid nucleation due to the initiation of an instantaneous local supersaturation of the precursor solution on the spinning substrate, resulting in a uniform seed layer for the subsequent perovskite crystallization step. ${ }^{[29]}$ Thus, the use of antisolvents together with solvent engineering for the formation of intermediary phase has become the mainstream for preparing solution-based perovskite materials, yielding high-performance PSCs. ${ }^{[31-33]}$ However, commonly used antisolvents such as chlorobenzene or toluene ${ }^{[29,30]}$ are environmentally harmful and highly toxic. While there have been proposals to replace these solvents with less toxic and more environmentally benign alternatives, ${ }^{[34,35]}$ the use of different antisolvent does not address the issue of important limitations in the scalability of the antisolvent assisted crystallization. ${ }^{[28]}$ In this process, the perovskite film quality critically depends on the time, volume and position of the application of the antisolvent. Inaccurate control of the dripping process will cause gradients in oversaturation of the precursor solution, leading to spatially inhomogeneous nucleation of the perovskite and deteriorate the resultant film quality.

There is considerable interest in the development of novel deposition methods for high quality perovskite films, where nucleation and crystallization of the perovskite films are controlled without the use of antisolvent. Different techniques to achieve this have been proposed, such as vacuum-flash assisted solution process (VASP), ${ }^{[36]}$ flash infrared annealing, ${ }^{[37]}$ etc. While high efficiency PSCs $(\approx 18 \%-20 \%)$ have been demonstrated, ${ }^{[36,37]}$ typically only one composition of the perovskite material is investigated. Due to increasing importance of mixed composition perovskite materials for high efficiency and improved stability PSCs, ${ }^{[9,36,38]}$ it is important to develop a simple, convenient, cost-effective, and reproducible technique applicable for the preparation of different perovskite materials. In this work, we introduce a novel perovskite growth process, in which the nucleation and crystallization processes are decoupled and, hence, independently controlled resulting in highly uniform nucleation sites for subsequent crystallization of perovskites. This is shown to be crucial for achieving high crystallinity in the perovskite films. Our method results in significant improvements in solar cell performance for three different compositions of mixed perovskite films, indicating general applicability to different perovskite materials.

We have developed a novel cryo-controlled nucleation technique, which effectively decouples the nucleation and crystallization phases and ensures the formation of a uniform seed layer for subsequent perovskite growth. The schematic diagrams shown in Figure 1a,b indicate the differences between the conventional spin-coating process and the proposed perovskite growth technique. The conventional 1-step process for a mixed perovskite precursor solution can result in non-uniform films with poor morphology due to coalescence of perovskite crystallites resulting from rapid solvent evaporation from a complex solution of the constituents with different solubilities. Figure 1a shows typical optical images of a mixed halide precursor and perovskite films $\left(\mathrm{Cs}_{0.05}\left(\mathrm{MA}_{0.17} \mathrm{FA}_{0.83}\right)_{0.95} \mathrm{~Pb}\left(\mathrm{I}_{0.84} \mathrm{Br}_{0.16}\right)_{3}\right)$ that we used in this work. The inhomogeneous formation of large crystallites is observed in the precursor film during the spinning process and a very non-uniform perovskite film is obtained after thermal annealing. Figure $1 \mathrm{~b}$ illustrates our proposed material growth method, which is a 4-step process as detailed below: (i) spin-coating of the precursors; (ii) cryogenic treatment; (iii) blow-dry process for the removal of the solvent; and (iv) thermal annealing. First, the precursors were spin-coated onto a glass/fluorine-doped tin oxide (FTO) $/ \mathrm{SnO}_{2}$ substrate. The spin-coating time was optimized to provide a uniform precursor film while preventing premature crystallization caused by excessive spinning due to the evaporation of a large amount of solvent. Second, the as-cast precursor film was dipped into a liquid nitrogen $\left(\mathrm{LN}_{2}\right)$ bath immediately. This treatment had two significant impacts on the precursor film. The rapid reduction in temperature induced abrupt freezing of the solvent consisting of a mixture of DMF (m.p. $-61{ }^{\circ} \mathrm{C}$ ) and DMSO (m.p. $19{ }^{\circ} \mathrm{C}$ ). The rapid solidification of the solvent at cryogenic temperature prevented chemical reaction and the coalescence of the precursors and, thereby, ensured the uniform distribution of the solutes. This phenomenon is demonstrated in Figure S1 of the Supporting Information, which shows the images of the precursor solution during the temperature reduction process. The rate of chemical reaction is described by the Arrhenius equation $k=A e^{-E_{a} /\left(k_{B} T\right)}$, where $k$ is the rate constant, $T$ is the absolute temperature, $A$ is a constant for chemical reaction that defines the rate due to the frequency of collisions in the correct orientation, $E_{\mathrm{a}}$ is the activation energy for the reaction, and $k_{\mathrm{B}}$ is the Boltzmann constant. The Arrhenius equation illustrates strong temperature dependence of the chemical reaction rate which was substantially reduced by immersing the substrate in the $\mathrm{LN}_{2}$ bath. If premature chemical reaction is not properly inhibited, large perovskite crystallites may be formed in the early stage and results in poor film morphology, which is irreversible even by introducing additional post-deposition treatments (e.g., thermal annealing). Third, upon removal from the $\mathrm{LN}_{2}$, the temperature of the substrate increased slowly to above the melting point of the solvent, which is estimated to be roughly in the range of $-50{ }^{\circ} \mathrm{C}$ in our case and the sample is subjected to a blow-dry process, using dry $\mathrm{N}_{2}$, to facilitate the removal of the residual solvents in the precursor film before thermal annealing. A supersaturated condition was induced in the solution resulting in the uniform precipitation of the 


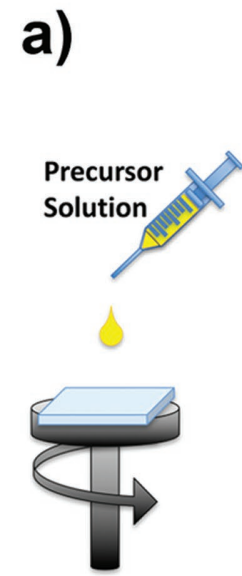

Spin Coating
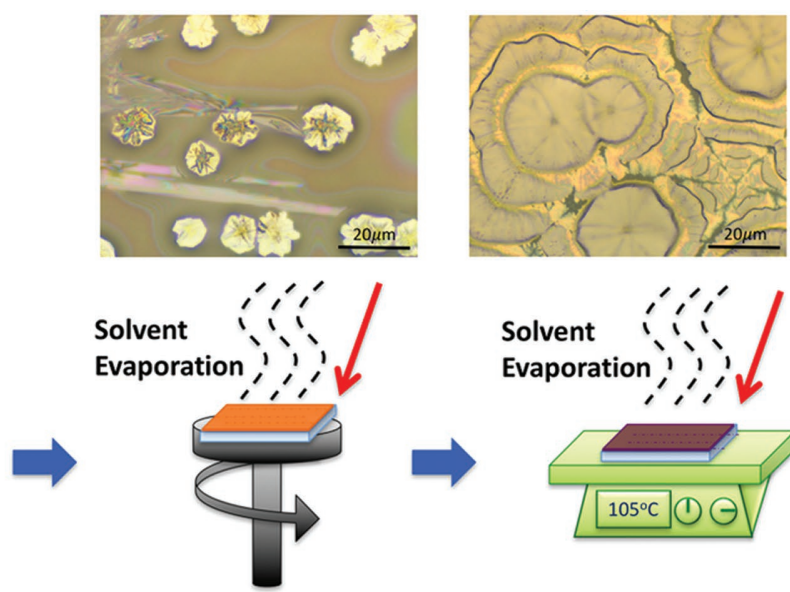

Spin Coating

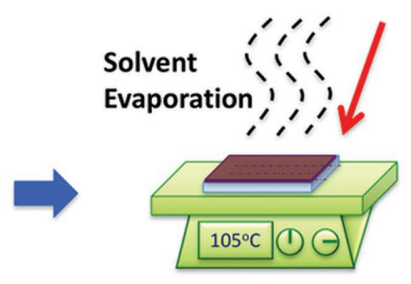

Thermal Annealing

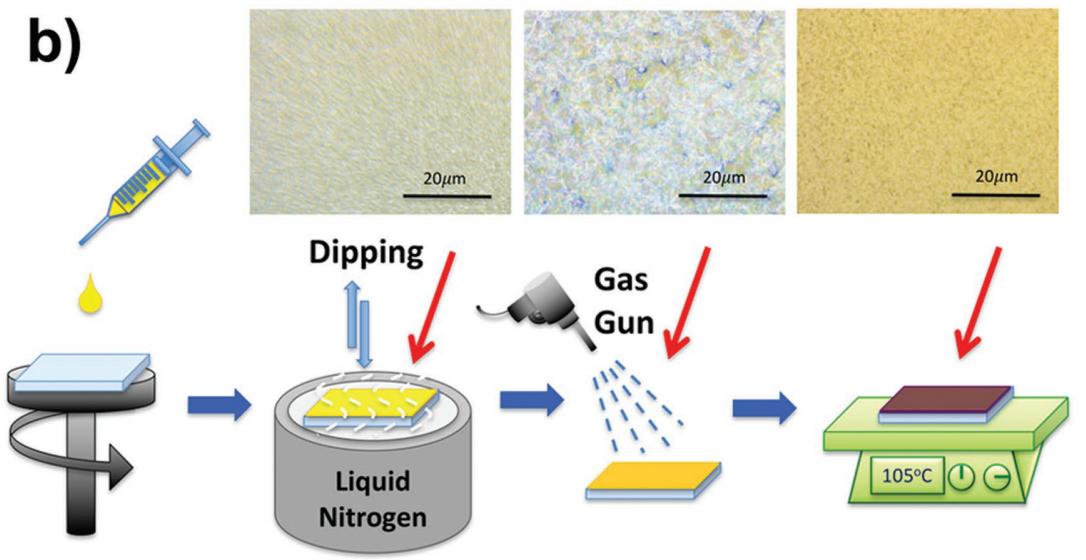

Spin Coating Cryogenic Treatment Blow Dry Thermal Annealing

precursors with large amounts of residual solvents were directly annealed, the precursors would have re-dissolved in the solvent and will nullify all the effects of the cryogenic treatment. The resultant perovskite films will become very rough with a high density of pinholes as in the case of films processed using the conventional technique. Another important attribute of the proposed cryo-controlled technique is the ease of scalability of the cryogenic dipping and the blow-drying steps to large area substrates with high uniformity. Finally, the sample was subjected to a thermal annealing treatment to completely convert the precursors into the perovskite material. Using this 4-step process, we were able to obtain very smooth precursor/perovskite films with excellent coverage over the entire substrate as observed from the insets in Figure 1b, which is very different from the films deposited using the conventional method as indicated in the insets of Figure 1a.

To investigate the applicability of the proposed cryogenic process to a variety of different compositions of mixed perovskites, we have conducted detailed characterizations on the following three types of perovskite films:

(1) Type A precursor/perovskite films$\mathrm{MA}_{x} \mathrm{FA}_{1-x} \mathrm{PbI}_{3}$ with the precursor recipe from Ding et al.; [39]

(2) Type $\mathrm{B}$ precursor/perovskite films$\mathrm{Cs}_{0.05}\left(\mathrm{MA}_{0.17} \mathrm{FA}_{0.83}\right)_{0.95} \mathrm{~Pb}\left(\mathrm{I}_{0.84} \mathrm{Br}_{0.16}\right)_{3}$ in which $\mathrm{Cs}$ and $\mathrm{Br}$ were incorporated into the precursor solution in addition to the basic elements in Type A films. It is known that the introduction of tertiary (FA/MA/Cs) cations and binary $(\mathrm{I} / \mathrm{Br})$ halide anions in Type B perovskite can significantly enhance its thermal stability and suppress the formation of photoinactive nonperovskite phase (yellow phase) in the resultant film, leading to enhanced performances for the PSCs; $; 9,38]$ and

(3) Type $\mathrm{C}$ precursor/perovskite films- $\mathrm{Cl}$ is incorporated to the Type B precursor.

Both the conventional and cryo-controlled growth processes were applied to all three types of films. For Type A films grown using the conventional process, it was observed that the crystallization of the perovskite

precursors over the entire substrate. It is important to point out that at this point the temperature of the substrate is still well below the temperature needed for chemical reactions, which prevents the coalescence of large pre-maturely formed perovskite crystallites. In addition, the blow-dry process is essential for rapid removal of residual solvents. If the films containing began in the early stage of spin-coating process resulting in a fast and short time window for the crystallization step. The ascast precursor film was gray in color, which yields a perovskite film with high roughness and poor coverage after the thermal annealing process. From Figure 1c, it is observed that there was a rapid change in the color of the Type A as-cast precursor film 

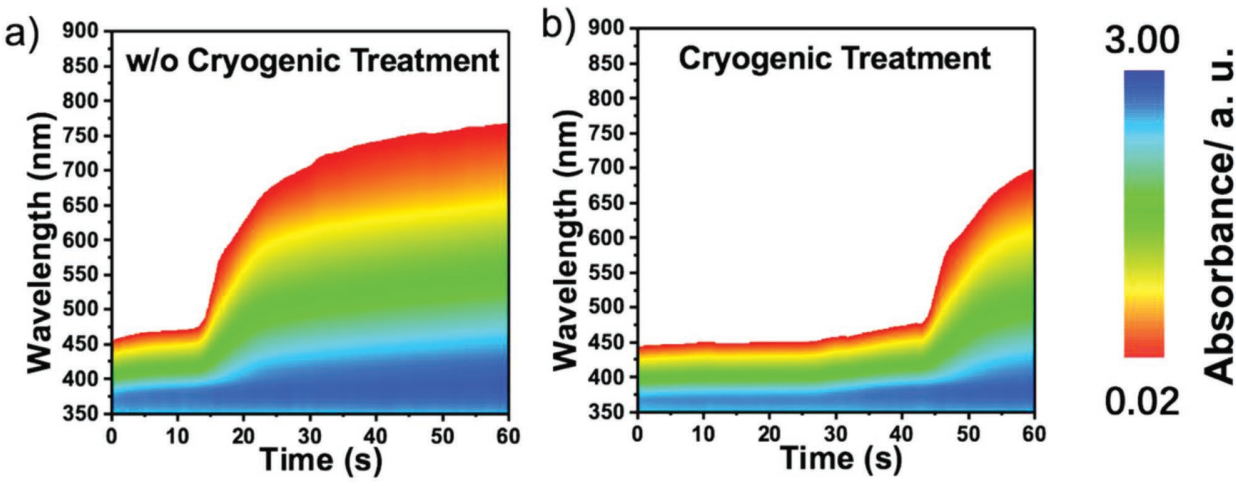

Figure 2. a,b) The results of dynamic absorption of the precursor film without (a) or with (b) the cryogenic treatment followed by the blow-dry process.

and, after thermal annealing, the resultant perovskite film had a very rough appearance. As for Type B and Type C precursor films, the time window was relatively longer compared to Type A precursor film. The as-cast precursor films were shiny at the beginning, but became rough and gray after $180 \mathrm{~s}$. Hence, the conventional perovskite growth method was not optimized for preparing Type B and C perovskites as it yielded grey films with very poor coverage as shown in the photos in Figure S2 of the Supporting Information. With the proposed cryogenic process, the crystallization rate was substantially lowered and the time window for the film formation was lengthened for all three perovskite compositions. From Figure 1c it is shown that the time window for film formation was significantly increased for Type A precursors. The cryogenic treatment was able to preserve the Type B and Type C precursor films in a shiny and smooth appearance for a longer time $(>15 \mathrm{~min})$ before carrying out the next processing step.

An in situ investigation of the 1-step spin-coating process of $\mathrm{MAPbX}_{3}(\mathrm{X}=\mathrm{I}, \mathrm{Br}, \mathrm{Cl})^{[40]}$ showed that the composition of the precursor solution has strong impact on the interaction between solvents and the solutes and thus alters the film casting dynamics during the spinning process, leading to the formation of ordered or disordered precursor solute phases. For most commonly used deposition recipes, the solvents tend to retain in the films and disrupt the precursor orders so that the quality of the resultant perovskite films may be influenced by a number of random factors, particularly for the processing conditions (e.g., concentration of solvents trapped in the glove box), resulting in diverse qualities for the perovskite materials even based on the same precursor formulation. Our proposed 4-step process is an effective strategy to reduce the impact caused by the solvent-solute interactions on the quality of the as-cast precursor films, which is particularly useful for mixed perovskites with high complexities in the composition. This is because the nucleation and crystallization phases are effectively decoupled during the perovskite growth.

The blow-dry process, similar to VASP ${ }^{[36]}$ and single gas quenching technique, ${ }^{[41,42]}$ relies on rapid residual solvent removal to control the film crystallization and improve uniformity. Adding the cryogenic treatment prior to blow-drying further enhances the decoupling of the nucleation and crystallization phases for the as-cast precursor films, yielding higher quality perovskite thin films after thermal annealing.
To investigate the role of these two steps in the film formation, we have conducted a dynamic absorption measurement on the as-cast precursor films treated by the blow-dry process alone and one prepared by the cryogenic technique followed by the blow-dry process. Figure 2 shows the comparison of the experimental results between these two types of samples based on Type B precursor films while the experimental results on Type A and Type C films are shown in Figure S3 of the Supporting Information. As observed in Figure 2, the unreacted pale-yellow precursor films absorbed photons between the range of 350 to $\approx 450 \mathrm{~nm}$. The increase in absorption beyond $450 \mathrm{~nm}$ indicates that chemical reactions had taken place between the precursors within the films leading to the formation of perovskite crystallites. It is clearly observed that the rate of conversion of the perovskite layer is more gradual for the sample treated with cryogenic process. Similar phenomena are also observed for the Type A and Type C samples (Figure S3, Supporting Information). The data show that chemical reactions between precursors and the evaporation of the residual solvents took place simultaneously. The low temperature ambient slows down the rate of precursor reaction and in the meantime the blow-dry process can effectively extract most of the residual solvent from the precursor films when the temperature increased, preventing the disordering of the precursor due to the melting of the residual solvents.

Retardation of the rate of chemical reactions by the cryogenic step in the process is also clear from the film morphology. Figure 3 shows the typical SEM images for the Type B films after each processing step: (i) as-cast precursor films with or without cryogenic treatment (Figure 3d,a, respectively); (ii) the precursor films after the blow-dry process (Figure 3b,e); and (iii) the final perovskite films after thermal annealing (Figure 3c,f). As observed in Figure 3a, substantial aggregation was observed for the as-cast precursor film without cryogenic treatment whereas a highly uniform precursor film was obtained for the cryogenically treated sample as shown in Figure 3d. After the blow-dry process, the cryogenically treated film exhibited excellent coverage (Figure 3e) compared to the control film which showed significant roughness and contained high concentration of pinholes (Figure 3b). Due to the substantial differences in the morphology of precursor films, it is not surprising that the resultant perovskite films exhibited tremendous differences in quality after thermal annealing. The control 


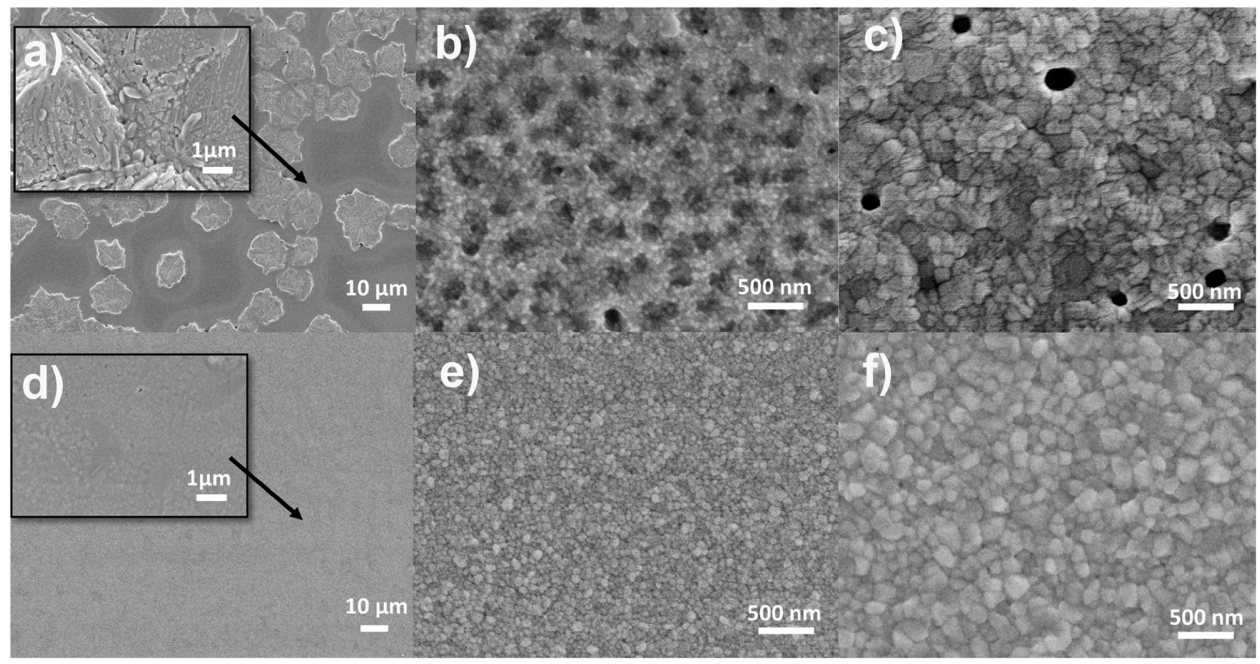

Figure 3. The morphology of the precursor/perovskite films prepared by the solution processes with or without cryo-controlled technique. a) The as-cast precursor film without cryogenic treatment, b) the precursor film without cryogenic treatment and after blow-dry process, c) the perovskite film obtained from thermally annealed precursor film in (b), d) the as-cast precursor film with cryogenic treatment, e) the precursor film with cryogenic treatment and after blow-dry process, and f) the perovskite film obtained from thermally annealed precursor film in (e).

perovskite film indicated poor coverage and high concentration of pinholes, as shown in Figure 3c, whereas the perovskite film grown by the proposed 4-step technique indicated excellent film morphology (Figure 3f).

It is known that the constituents used in the precursors greatly affect the dynamics of perovskite conversion. ${ }^{[40]}$ It is found that the average grain size of Type A perovskite film (Figure S4c, Supporting Information) is substantially smaller compared to that of Type B films (Figure 3f) since the rate of crystallization of Type A film is much faster than the Type B film during the thermal annealing stage leading to significantly shorter crystallization time. However, large grain size is desirable for high-performance PSCs, since films with larger grain size have fewer grain boundaries and lower trap density. ${ }^{[27]}$ In our previous work, we have shown that the crystallization time is substantially lengthened when the concentration of $\mathrm{Cl}$ in the precursor solution increases and the perovskite grain size is proportional to the crystallization time. ${ }^{[13]}$ Furthermore, Bouchard et al. ${ }^{[4]}$ utilized synchrotron X-ray diffraction microscopy to obtain direct evidence for chlorine-induced preferential crystalline orientation leading to the growth of larger perovskite crystallites on $\mathrm{TiO}_{2}$. Thus, we considered modification of the precursor solution composition by adding $\mathrm{Cl}$ to further increase the grain size of the perovskite (Type C). Our experimental data show that the crystallization rates for the three types of perovskites in this work are: Type A>Type B>Type C, with a strong impact on the average grain size as observed in the corresponding films: Type A<Type B<Type C, as clearly shown in the SEM images in Figure $3 f$, and Figure S4c,f,I (Supporting Information). Figure S4 of the Supporting Information also compares three types of resultant perovskite films prepared by the conventional process (direct thermal annealing of the as-cast precursor films), blow dry process followed by thermal annealing and the proposed 4-step cryogenic process. It is noted that introduction of the blow-dry process before thermal annealing can yield perovskite films with enhanced coverage compared to the samples prepared by direct thermal annealing of the as-cast precursor films. However, pinholes likely appear for the type of perovskite with fast crystallization rates. This observation is consistent to the results of dynamic absorption shown in Figure 2 and Figure S3 (Supporting Information), which clearly indicate that samples treated with single blow-dry process have shorter crystallization time window and the precursors start to react right after the spin-coating process, increasing the chance of premature crystallization. On the contrary, all three types of multication mixed halide perovskites grown by the proposed 4-step technique exhibited substantial improvements in the film uniformity and coverage compared to the counterparts grown without cryogenic treatment. The results reflect the effectiveness of the proposed technique for preparing a wide range of organometal halide perovskite materials with excellent morphology. Moreover, the cryogenic step is particularly useful for the future manufacturing production lines as it can preserve the precursor films as long as the next processing step is ready. The perovskite film quality prepared by the blow-dry process and cryogenic 4-step process are compared by different characterization techniques and the results are shown in the following paragraphs.

The X-ray diffraction (XRD) results provide further insight into the evolution of Type B film at different stages of material processing. We have investigated two different films: i) the control film (without cryogenic treatment) with the results shown in curves 1, 3, and 5 of Figure 4a; and ii) Type B film fabricated with the proposed 4-step technique and the results are shown in curves 2, 4, and 6 of Figure 4a. From the data, it is observed that no crystal phase in the as-cast precursor films can be resolved (curves 1 and 2) as the precursors were still embedded in the residual solvents after spin-coating process without any preferential crystal orientation for both samples. When the residual solvents were being extracted with the aid of the blow-dry process (curves 3 and 4), a number of crystal phases can be detected. It is interesting to point out that the 

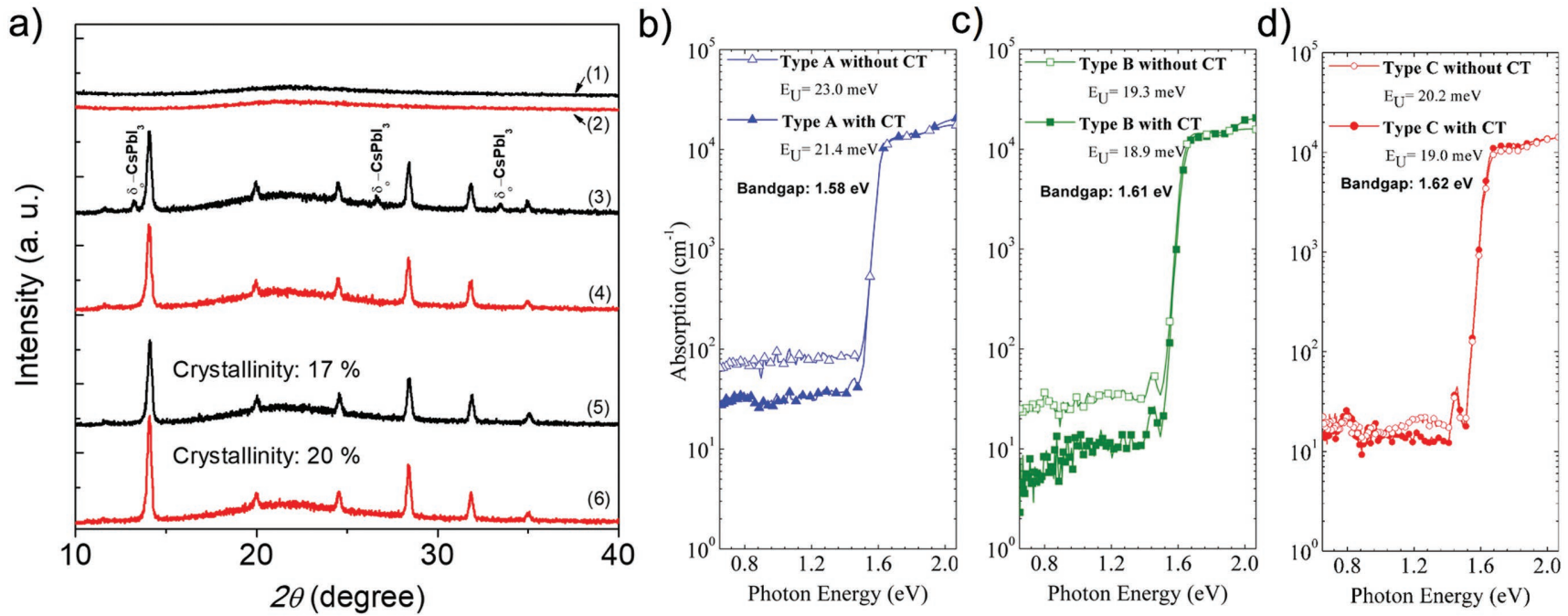

Figure 4. a) The XRD patterns for Type B precursor/perovskite films at different processing steps. The control results are plotted in black (curves $1,3,5$ ) and the results obtained from the film prepared by the cryo-controlled technique are plotted in red (curve 2, 4, 6). Curves 1 and 2: as-cast precursor films, Curves 3, 4: after blow-dry process, Curves: 5, 6: after blow-dry process and thermal annealing. b-d) The PDS spectra of Type A (b), Type B (c), and Type C (d) perovskite films prepared by solution processes with and without cryo-controlled technique (CT).

yellow phase $\left(13.2^{\circ}, 26.6^{\circ}\right.$, and $\left.33.8^{\circ}\right)$ of $\mathrm{CsPbI}_{3}\left(\delta_{\left.\mathrm{O}^{-}-\mathrm{CsPI}_{3}\right)^{[44]}}\right.$ in addition to the characteristic perovskite phases $\left(14.1^{\circ}\right.$, $19.9^{\circ}, 24.5^{\circ}, 28.4^{\circ}, 31.8^{\circ}$, and $35^{\circ}$ ) were observed from curve 3 obtained from the control sample whereas no yellow phase of $\mathrm{CsPI}_{3}$ was detected from the sample treated with cryogenic process (curve 4). The results are indicative of the impact of the cryogenic process, which can suppress the formation of undesired phases in the precursor film during the blow-dry process and it is believed that the reduction in the reaction rate of the precursors after the cryogenic treatment had led to the formation of ordered precursor solute phases. ${ }^{[40]}$ After the complete conversion of the perovskite material from the precursors, XRD peaks $\left(14.1^{\circ}, 19.9^{\circ}, 24.5^{\circ}, 28.4^{\circ}, 31.8^{\circ}\right.$, and $\left.35^{\circ}\right)$ were observed from curves 5 and 6 of Figure 4a, which are consistent with the literature. ${ }^{[38]}$ From the experimental data, we investigated the crystallinity of the two films based on the ratio of the perovskite peaks and the background XRD signal. It is observed that despite the disappearance of the yellow phase for the control sample after thermal annealing, the crystallinity of the control film is still substantially lower compared to the sample with cryogenic treatment. The XRD results for Types A and C perovskite samples prepared with and without cryogenic treatment are shown in Figure S5 (Supporting Information) and the results are found to be consistent with the discussions above. Our results agree well with the recent work of Ren et al., ${ }^{[45]}$ who found that the incorporation of low temperature $\left(0^{\circ} \mathrm{C}\right)$ antisolvent during the spinning process can stimulate homogeneous nuclei growth and thus improve the crystallinity of the perovskite films, yielding an average PCE of $18.4 \%$ for $\left(\mathrm{FAPbI}_{3}\right)_{0.85}\left(\mathrm{MAPbBr}_{3}\right)_{0.15}$ based PSCs.

We further investigated the impact of the composition of the precursors on the crystallinity of the perovskite film grown using the 4-step processes. From the XRD results it is observed that the degree of crystallinity is improved from $\approx 20 \%$ (Type A and Type B perovskite) to $85 \%$ (Type C perovskite), which is due to the further increase in the grain size for Type $\mathrm{C}$ perovskite layer achieved by slowing down the crystallization process with the incorporation of $\mathrm{Cl}$. Furthermore, several groups have pointed out that incorporation of $\mathrm{Cl}$ affects the morphological evolution of perovskite growth, leading to preferential crystallographic orientations, which is beneficial for efficient charge transfer. ${ }^{[46,47]}$ Their findings are also corroborated by our XRD data for the Type C perovskite, as indicated in Figure S5 of the Supporting Information.

We also investigated the defects in the films with and without cryogenic treatment using photothermal deflection spectroscopy (PDS). The technique demonstrates high sensitivity (order of $10^{-4}$ ) in absorption characterization as it can detect the changes in the thermal state of the samples due to the nonradiative relaxation of photoexcited carriers. It has been commonly applied to characterize the energetic disorder as the exponential decay of the absorption below the bandgap with a characteristic Urbach energy $E_{U}{ }_{U}^{[48]}$ and analyze the electronic defects in amorphous and organic semiconductors. ${ }^{[49,50]}$ In this work, we have performed PDS to examine the density of the bandgap states of the three types of perovskite films with and without cryogenic treatment, as shown in Figure $4 \mathrm{~b}-\mathrm{d}$. From the results, the conduction band edges are found to be $\approx 1.58,1.61$, and $1.62 \mathrm{eV}$ from valence band edge for the Type A, Type B, and Type C perovskite films, respectively, which is in excellent agreement with absorbance spectra of the films as shown in Figure S6 of the Supporting Information. The shallow traps of the films can be characterized by the magnitude of $E_{U}$, which reveals the steepness of the band tail states located at the conduction band edge. ${ }^{[51]} \mathrm{A}$ smaller $E_{U}$ stipulates a lower density of shallow traps and thus these data are useful figure-of-merits for the films. The $E_{U}$ values for the three types of perovskites with or without cryogenic treatment, as listed in Table 1, clearly show that the cryogenic treatment substantially suppresses the formation of both the shallow and deep traps for all three types of perovskite films. 
Table 1. The summary of parameters of devices and films for different perovskite compositions with and without cryogenic treatment. The values of photovoltaic parameters are averaged from ten devices and the highest values are indicated in the bracket.

\begin{tabular}{lccccccc}
\hline Type & Cryogenic & $V_{\mathrm{OC}}[\mathrm{V}]$ & $J_{\mathrm{SC}}\left[\mathrm{mA} \mathrm{cm}{ }^{-2}\right]$ & $\mathrm{FF}$ & PCE $[\%]$ & $E_{U}[\mathrm{meV}]$ & $\tau[\mathrm{ns}]$ \\
\hline A & Without & $1.04 \pm 0.05(1.10)$ & $21.1 \pm 0.7(20.4)$ & $0.58 \pm 0.05(0.69)$ & $12.7 \pm 1.5(15.3)$ & 23.0 & $13 \pm 1$ \\
& With & $1.07 \pm 0.01(1.07)$ & $22.9 \pm 0.4(23.9)$ & $0.72 \pm 0.02(0.74)$ & $17.8 \pm 0.7(18.9)$ & 21.4 & $22 \pm 1$ \\
B & Without & $1.08 \pm 0.02(1.10)$ & $22.4 \pm 1.3(23.1)$ & $0.65 \pm 0.03(0.67)$ & $15.8 \pm 1.0(17.1)$ & 19.3 & $89 \pm 2$ \\
& With & $1.11 \pm 0.01(1.13)$ & $22.0 \pm 0.8(22.5)$ & $0.77 \pm 0.02(0.79)$ & $18.8 \pm 0.7(20.1)$ & 18.9 & $288 \pm 5$ \\
C & Without & $1.10 \pm 0.01(1.11)$ & $22.0 \pm 0.3(22.3)$ & $0.74 \pm 0.03(0.77)$ & $18.1 \pm 0.7(19.0)$ & 20.2 & $460 \pm 4$ \\
& With & $1.13 \pm 0.01(1.14)$ & $23.0 \pm 0.5(23.5)$ & $0.79 \pm 0.01(0.80)$ & $20.5 \pm 0.6(21.4)$ & 19.0 & $690 \pm 5$ \\
\hline
\end{tabular}

To further investigate the film quality and defect density time resolved photoluminescence (TRPL) measurements were performed, and the obtained results are shown in Figure 5a. All data can be well fitted to the first order exponential decay function and the carrier lifetimes, $\tau$, can be determined from the fitting. The values of $\tau$ for the three types of perovskites with or without cryogenic treatment are indicated in Table 1. For all perovskite compositions, cryogenic treatment results in longer lifetimes, which is consistent with expected lower trap density based on improved crystallinity (XRD), larger grain size (SEM), and lower density of gap states (PDS). A longer lifetime is a clear indication of reduction in carrier recombination in the perovskite films due to the enhanced film quality, which is beneficial for photovoltaic performance.

We also performed low-frequency noise (LFN) measurements on the three types of perovskite, which provides further evidence on the impact of the cryo-treatment on the crystallinity of the perovskite films. The LFN is a ubiquitous phenomenon, which is observed in all semiconductor devices including the PSCs. The noise power spectral density (PSD) typically is expressed in a functional form of $S(f) \propto 1 / f^{\gamma}$ where $\gamma \approx 1$. It has been widely demonstrated in semiconductor optoelectronic and electronic devices that the LFN in the device conductance originates from the random trapping and detrapping of the carriers by localized defect states in the material. ${ }^{[52,53]}$ The random trapping and detrapping events of the carriers by a single defect will result in the corresponding fluctuation in the conductance of the device in the form of a random telegraph noise with the noise PSD given by

$S(f)=\frac{A \tau}{1+4 \pi^{2} f^{2} \tau^{2}}$

where $A$ is a proportionality constant and $\tau$ is the fluctuation time constant. It has been showed that LFN of PSCs arises from a thermally activated trapping and detrapping process, ${ }^{[17]}$ hence the fluctuation time constant is given by $\tau=\tau_{0} \exp \left(\frac{E}{k_{\mathrm{B}} T}\right)$, where $\tau_{0}$ is of the order of the inverse phonon frequency of the order $10^{-12} \mathrm{~s}, E$ is the activation energy of the trap, $k_{\mathrm{B}}$ is the Boltzmann constant, and $T$ is the absolute temperature. Since the individual trapping and detrapping events due to different trap states are statistically independent, the overall current noise PSD, $S_{I}(f)$, for a large device with large number of localized states can be expressed as

$\frac{S_{I}(f)}{I^{2}}=A \int_{x} \int_{y} \int_{z} \int_{E} N_{T}(E, x, y, z) \frac{\tau}{1+4 \pi^{2} f^{2} \tau^{2}} \mathrm{~d} E \mathrm{~d} z \mathrm{~d} \gamma \mathrm{d} x$

where $N_{\mathrm{T}}$ is the trap density and $I$ is the DC current applied to the device. Based on the thermal activation model for the LFN, the trap density $N_{\mathrm{T}}$ can be expressed as

$$
N_{\mathrm{T}}\left(E_{\mathrm{p}}\right) \approx \frac{4 C f}{k_{\mathrm{B}} T} \frac{S_{I}(f)}{I^{2}}=\frac{4 C f}{k_{\mathrm{B}} T} \frac{S_{V}(f)}{V^{2}}
$$
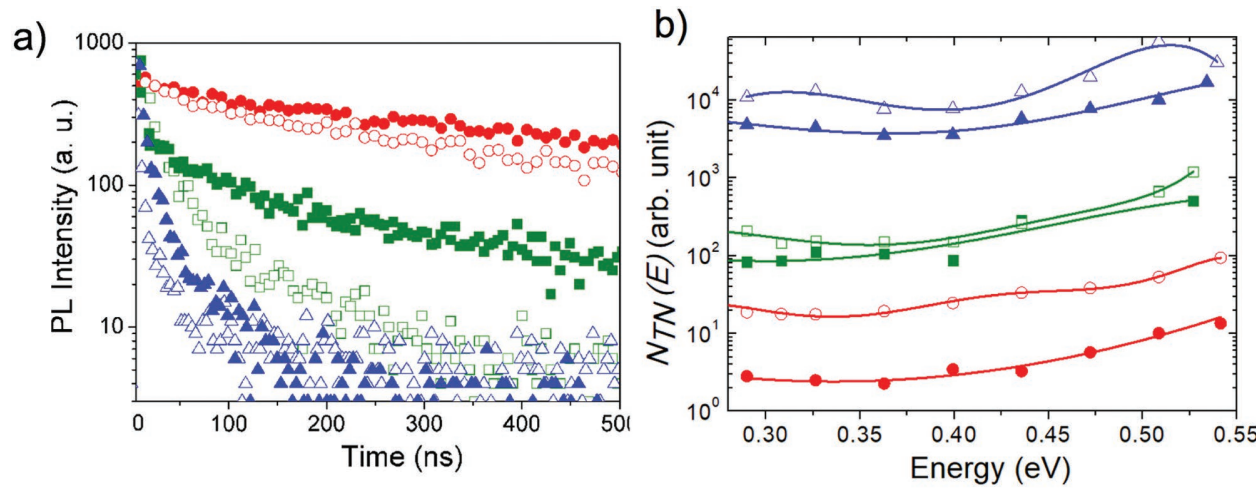

$\triangle$ Type A without CT

- Type A with CT

\section{Type B without CT Type C without CT Type B with CT \\ - Type C with CT}

Figure 5. a) TRPL signals and b) LFN spectra for three types of perovskite film prepared by the solution processes with and without cryo-controlled technique (CT). 
where $C$ is a proportionality constant and the Lorentzian peaks sharply at $E_{\mathrm{p}}=-k_{\mathrm{B}} T \ln \left(\omega \tau_{0}\right)$. To pinpoint the impact of material growth technique on the defect density of perovskites, we have performed the LFN measurement on the samples with a resistive structure (FTO/perovskite/Au), which not only characterizes the bulk of the perovskite layer but also the perovskite/FTO and perovskite/Au interfaces as well. Based on Equation (3) above the normalized trap densities, $N_{\mathrm{TN}}(E)$ were computed for the different samples by adopting a unity value for $C$ and the results are shown in Figure $5 \mathrm{~b}$. The results demonstrate substantial decrease in the trap density for all types of perovskite samples prepared by the 4-step growth technique compared to their counterparts, which were not treated with the cryogenic process, in agreement with PDS and TRPL results. In addition, an obvious trend of reduction in trap density from the samples of Type A to Type C is observed in Figure 5b. It is believed that perovskite films with larger gain size contain lower concentration of grain boundaries, which can substantially reduce the trap density in the bulk of the perovskite films and between the material interfaces.

The photovoltaic performance of the PSCs based on the three types of perovskite films were characterized in detail. From the photovoltaic parameters summarized in Table 1 , it is clearly observed that cryogenic treatment results in significant enhancements in open-circuit voltage $\left(V_{\mathrm{OC}}\right)$, and fill factor $(\mathrm{FF})$ for all types of perovskites. Substantial improvements in shortcircuit current density $\left(U_{\mathrm{SC}}\right)$ for Type A and Type $\mathrm{C}$ devices and no significant change in $J_{\text {SC }}$ for Type B devices are observed. Overall, the cryo-controlled grown PSCs have higher PCEs compared to their counterparts without using the cryocontrolled process. Furthermore, an ascending trend in the PCE is observed for the different types of PSCs: Type A<Type $\mathrm{B}<$ Type $\mathrm{C}$, which strongly correlates with the experimental results obtained above, since Type $\mathrm{C}$ perovskite films have larger grain size, higher crystallinity, and lower trap density.

The representative current-voltage $(I-V)$ curves and external quantum efficiency (EQE) spectra for the three types of PSCs prepared by the cryo-controlled process are plotted in Figures S7 and S8 of the Supporting Information. We have achieved a champion device with PCE of $21.4 \%$ with $V_{\mathrm{OC}}=1.14 \mathrm{~V}$, $J_{\mathrm{SC}}=23.5 \mathrm{~mA} \mathrm{~cm}^{-2}$ and a high FF of 0.80 from Type C PSC (Figure 6a). It is noteworthy that our PSCs, based on a compact $\mathrm{SnO}_{2}$ film as the electron transport layer (ETL), have different extents of hysteresis as shown in Figure S7 of the Supporting Information. The hysteresis index (HI) is defined as $\left.\mathrm{HI}=\left[P_{\text {max }, \text { reverse }} / P_{\max , \text { forward }}\right]-1\right),{ }^{[54]}$ where $P_{\max }$ is the maximum power points on the reverse and the forward scan. Among the three types of perovskites, Type C PSC has the lowest HI (0.18) compared to Type A (0.30) and Type B (0.32) PSCs, which is attributed to the improved film quality of Type $\mathrm{C}$ perovskite.

The residual hysteresis in our devices after optimization of perovskite quality is attributed to the surface traps, which is also consistent with slow increase of photocurrent observed in current-time $(I-t)$ scan shown in Figure S9 of the Supporting Information. ${ }^{[55]}$ It was also reported that the hysteresis is related to the energy level alignment at ETL/perovskite interface, ${ }^{[56,57]}$ which in turn is affected by the presence of deep trap states. ${ }^{[57]}$ In addition to the reduction in the interface trap density, improvements in the conductivity of $\mathrm{SnO}_{2}$ may also contribute to reduced hysteresis. ${ }^{[58]}$ a)

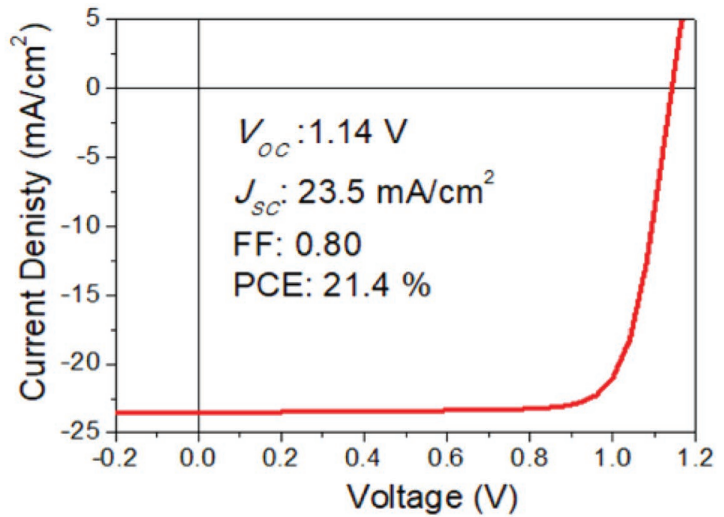

b)

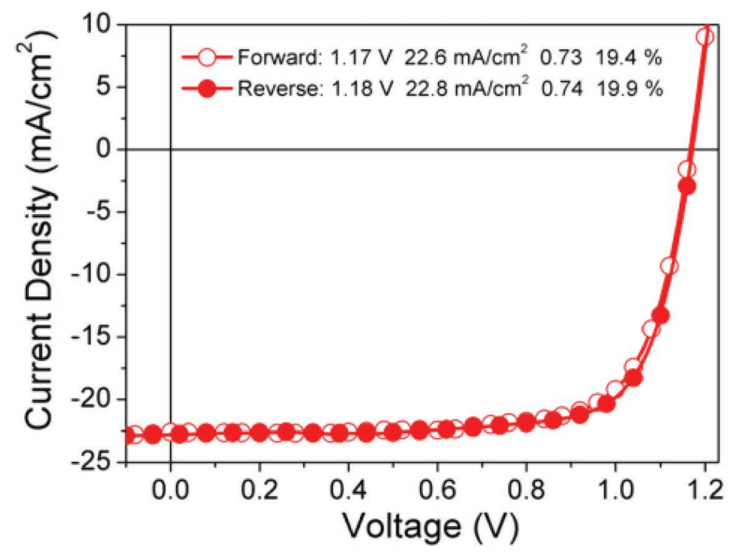

c)

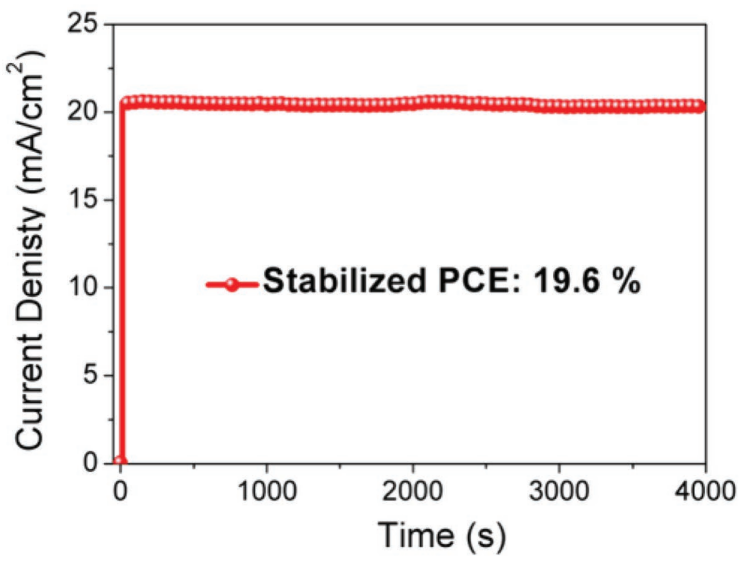

Figure 6. a) The $I-V$ characteristics of a PSC with the champion PCE. b) The typical reverse and forward $I-V$ scan and c) $I-t$ scan of the optimized PSCs.

Since the observed behaviors (hysteresis and slow stabilization dynamics) are consistent with the presence of interface traps rather than bulk traps in the perovskite layer, ${ }^{[55]}$ it is possible that the performance could be further improved by optimizing ETL deposition as well as the optimization of the interface between the ETL and the perovskite layer - an effect analogous to PSCs with mesoscopic ETL typically demonstrate smaller $I-V$ hysteresis. ${ }^{[24]}$

We have optimized the ETL/perovskite interface by incorporating a layer of $\mathrm{SnO}_{2}$ nanoparticles $\left(\mathrm{SnO}_{2}-\mathrm{NPs}\right)$ on top of the compact $\mathrm{SnO}_{2}\left(\mathrm{c}-\mathrm{SnO}_{2}\right)$ layer and the obtained $I-V$ curves shows significant reduction in hysteresis (Figure 6b). We have conducted atomic force microscopy on different types of ETL layers 
(c-SnO $\mathrm{Sn}_{2}$ and $\mathrm{c}-\mathrm{SnO}_{2} / \mathrm{SnO}_{2}-\mathrm{NPs}$ ) and the perovskite film (Type C) coated on the corresponding types of ETL. The results, as shown in Figure S10 of the Supporting Information, indicate a slight increase in surface roughness of the ETL (RMS from 14 to $33 \mathrm{~nm}$ ) and perovskite (RMS from 29 to $30 \mathrm{~nm}$ ) when $\mathrm{SnO}_{2}$ NPs were deposited on the $\mathrm{c}-\mathrm{SnO}_{2}$. It is interesting to note that no obvious change in perovskite morphology is found after introducing an extra layer of $\mathrm{SnO}_{2}-\mathrm{NPs}$, which is possibility due to the low concentration of $\mathrm{SnO}_{2}$-NPs in our recipe. We have also compared the TRPL signal on the samples with the structure of Quartz/c-SnO $2 /$ perovskite and quartz/c- $\mathrm{SnO}_{2} / \mathrm{SnO}_{2}$ NPs/perovskite. The results are plotted in Figure S11 of the Supporting Information. The effectiveness of electron transport between perovskite and ETL can be reflected by the quenching rate of the PL intensity. ${ }^{[59]}$ It is observed that the decay of TRPL signal is accelerated for the sample of quartz/c- $\mathrm{SnO}_{2} / \mathrm{SnO}_{2}$ $\mathrm{NPs} /$ perovskite compared to the sample of quartz/c-SnO $\mathrm{S}_{2}$ perovskite, indicating a more efficient electron transfer from the perovskite to the ETL composed of c- $\mathrm{SnO}_{2} / \mathrm{SnO}_{2}-\mathrm{NPs}$. The enhancement of carrier transports and reduction in trap density at the interface between the layer of ETL and perovskite in the PSC are believed to be the factors for reducing $I-V$ hysteresis.

The $I-t$ scan of the device under 1 sun illumination has been measured for $\mathrm{c}-\mathrm{SnO}_{2}$ based and $\mathrm{c}-\mathrm{SnO}_{2} / \mathrm{SnO}_{2}$-NPs based devices and both devices show good stability over a long period of time. It is noteworthy that the $\mathrm{c}-\mathrm{SnO}_{2}$ based devices in this work usually require a longer photocurrent stabilization time compared to $\mathrm{c}-\mathrm{SnO}_{2} / \mathrm{SnO}_{2}$-NPs based devices (compare Figure S9, Supporting Information with Figure 6c). Meanwhile light soaking effect is observed for $\mathrm{c}-\mathrm{SnO}_{2}$ based devices but not for $\mathrm{c}-\mathrm{SnO}_{2} / \mathrm{SnO}_{2}$-NPs based devices. It is speculated that the trap filling action at $\mathrm{c}-\mathrm{SnO}_{2}$ interface is accounted for the observed behaviors ${ }^{[55,60]}$ and their effects can be alleviated by optimizing the c-SnO 2 /perovskite interface with $\mathrm{SnO}_{2}$-NPs. The correlation between the defect density and the quality of $\mathrm{SnO}_{2}$ ETL will be systematically investigated in the coming works.

The scalability of PSCs is one of the concerns for commercialization. We have applied our proposed technique to prepare PSCs with larger area. The designed layout of the solar module is shown in Figure S12a,b of the Supporting Information, in which three devices are connected in series with a designated illumination area of $2.73 \mathrm{~cm}^{2}$. A PCE of $15.2 \%$ with a $V_{\mathrm{OC}}$ $3.35 \mathrm{~V}, J_{\mathrm{SC}} 18.8 \mathrm{~mA} \mathrm{~cm}{ }^{-2}$, and 0.72 fill factor can be achieved (Figure S12c, Supporting Information).

A novel 4-step cryo-controlled method for the deposition of high quality mixed perovskite films without the use of antisolvents has been demonstrated. This method results in increased grain size and crystallinity and lower defect density for all three different perovskite compositions investigated. Thus, the method has universal applicability and it is readily scalable to larger device areas. The improved film quality and consequently increased PCE result from decoupling of the nucleation and crystallization phases during the conversion of perovskite from precursors. Processing at cryogenic temperature suppresses premature reactions of the precursors and prevents premature coalescence of nuclei into large crystallites, enabling uniform film formation following the blow-drying and annealing steps. The method is of particular interest for Cs-containing perovskite formulations, since it is shown to successfully suppress the formation of the undesirable yellow phase $\delta$-CsPbI 3 . Using this method, a champion PCE of $21.4 \%$ with a $V_{\mathrm{OC}}=1.14 \mathrm{~V}, J_{\mathrm{SC}}=23.5 \mathrm{~mA} \mathrm{~cm}{ }^{-2}$, and $\mathrm{FF}=0.80$ can be achieved for optimized perovskite composition.

\section{Supporting Information}

Supporting Information is available from the Wiley Online Library or from the author.

\section{Acknowledgements}

A.N. and Z.W.R. contributed equally to this work. This work was supported by Nazarbayev University Grant Award No. 090118FD5326, the targeted Program BR05236524, the Seed Funding for Strategic Interdisciplinary Research Scheme of the University of Hong Kong, RGC GRF (Grant Nos. 15204515 and 15246816), and Shenzhen Science and Technology Commission (Project No. JCY)20170818141216288). Special thanks are given to Prof. Jianhua Hao and Dr. Gongxun Bai for professional assistance in TRPL measurement.

\section{Conflict of Interest}

The authors declare no conflict of interest.

\section{Keywords}

blow-dry process, cryogenic treatment, crystallization, nucleation, perovskite solar cells, residual solvents

Received: July 10, 2018

Revised: August 4, 2018

Published online: September 12, 2018

[1] A. Kojima, K. Teshima, Y. Shirai, T. Miyasaka, J. Am. Chem. Soc. 2009, 131, 6050.

[2] National Renewable Energy Laboratory, Best research-cell efficiencies, https://www.nrel.gov/pv/assets/images/efficiencychart-20180716.jpg (accessed: August 2018).

[3] J.-H. Im, C.-R. Lee, J.-W. Lee, S.-W. Park, Nanoscale 2011, 3, 4088.

[4] G. E. Eperon, S. D. Stranks, C. Menelaou, M. B. Johnston, L. M. Herz, H. J. Snaith, Energy Environ. Sci. 2014, 7, 982.

[5] S. D. Stranks, S. D. Stranks, G. E. Eperon, G. Grancini, C. Menelaou, M. J. P. Alcocer, T. Leijtens, L. M. Herz, A. Petrozza, H. J. Snaith, Science 2013, 342, 341.

[6] C. C. Stoumpos, C. D. Malliakas, M. G. Kanatzidis, Inorg. Chem. 2013, 52, 9019.

[7] W.-J. Yin, J.-H. Yang, J. Kang, Y. Yan, S.-H. Wei, J. Mater. Chem. A 2015, 3, 8926.

[8] O. D. Miller, E. Yablonovitch, S. R. Kurtz, IEEE J. Photovoltaics 2012, 2, 303.

[9] Q. Dong, F. Liu, M. K. Wong, H. W. Tam, A. B. Djurišić, A. Ng, C. Surya, W. K. Chan, A. M. C. Ng, ChemSusChem 2016, 9, 2597.

[10] W. Chen, Y. Wu, J. Fan, A. B. Djurišić, F. Liu, H. W. Tam, A. Ng, C. Surya, W. K. Chan, D. Wang, Z.-B. He, Adv. Energy Mater. 2018, 8, 1703519.

[11] A. B. Djurišić, F. Z. Liu, H. W. Tam, M. K. Wong, A. Ng, C. Surya, W. Chen, Z. B. He, Prog. Quantum Electron. 2017, 53, 1. 
[12] Z. H. Bakr, Q. Wali, A. Fakharuddin, L. Schmidt-Mende, T. M. Brown, R. Jose, Nano Energy 2017, 34, 271.

[13] C. Liu, R. Zhu, A. Ng, Z. Ren, S. H. Cheung, L. Du, S. K. So, J. A. Zapien, A. B. Djurišić, D. L. Phillips, C. Surya, J. Mater. Chem. A 2017, 5, 15970.

[14] K. Li, Y. Zhang, H. Zhen, H. Wang, S. Liu, F. Yan, Z. Zheng, J. Mater. Chem. A 2017, 5, 969.

[15] Z. Ren, J. Zhou, Y. Zhang, A. Ng, Q. Shen, S. H. Cheung, H. Shen, K. Li, Z. Zheng, S. K. So, A. B. Djurišić, C. Surya, Sol. Energy Mater. Sol. Cells 2018, 179, 36

[16] A. B. Djurišić, F. Liu, A. M. C. Ng, Q. Dong, M. K. Wong, A. Ng, C. Surya, Phys. Status Solidi RRL 2016, 10, 281.

[17] Q. Shen, A. Ng, Z. Ren, H. C. Gokkaya, A. B. Djurišić, J. A. Zapien, C. Surya, ACS Appl. Mater. Interfaces 2018, 10, 371.

[18] F. Liu, Q. Dong, M. K. Wong, A. B. Djurišić, A. Ng, Z. Ren, Q. Shen, C. Surya, W. K. Chan, J. Wang, A. M. C. Ng, C. Liao, H. Li, K. Shih, Adv. Energy Mater. 2016, 6, 1502206.

[19] Z. Ren, A. Ng, Q. Shen, H. C. Gokkaya, J. Wang, L. Yang, W.-K. Yiu, G. Bai, A. B. Djurišić, W. W. Leung, J. Hao, W. K. Chan, C. Surya, Sci. Rep. 2014, 4, 6752.

[20] J. Burschka, N. Pellet, S.-J. Moon, R. Humphry-Baker, P. Gao, M. K. Nazeeruddin, M. Grätzel, Nature 2013, 499, 316.

[21] J. Das, A. S. Subbiah, N. Mahuli, R. Singh, S. K. Sarkar, Energy Technol. 2017, 5, 1807.

[22] C. Momblona, O. Malinkiewicz, C. Roldán-Carmona, A. Soriano, L. Gil-Escrig, E. Bandiello, M. Scheepers, E. Edri, H. J. Bolink, APL Mater. 2014, 2, 081504.

[23] G.-X. Liang, P. Fan, J.-T. Luo, D. Gu, Z.-H. Zheng, Prog. Photovolt.: Res. Appl. 2015, 23, 1901.

[24] A. Ng, Z. Ren, Q. Shen, S. H. Cheung, H. C. Gokkaya, G. Bai, J. Wang, L. Yang, S. K. So, A. B. Djurišić, W. W. Leung, J. Hao, W. K. Chan, C. Surya, J. Mater. Chem. A 2015, 3, 9223.

[25] Q. Chen, H. Zhou, Z. Hong, S. Luo, H.-S. Duan, H.-H. Wang, Y. Liu, G. Li, Y. Yang, J. Am. Chem. Soc. 2014, 136, 622.

[26] M. K. Wong, F. Liu, C. S. Kam, T. L. Leung, H. W. Tam, A. B. Djurišić, J. Popović, H. Li, K. Shih, K.-H. Low, W. K. Chan, W. Chen, Z. He, A. Ng, C. Surya, Chem. Mater. 2017, 29, 9946.

[27] A. Ng, Z. Ren, Q. Shen, S. H. Cheung, H. C. Gokkaya, S. K. So, A. B. Djurišić, Y. Wan, X. Wu, C. Surya, ACS Appl. Mater. Interfaces 2016, 8, 32805.

[28] M. Konstantakou, D. Perganti, P. Falaras, T. Stergiopoulos, Crystals 2017, 7, 291

[29] M. Xiao, F. Huang, W. Huang, Y. Dkhissi, Y. Zhu, J. Etheridge, A. Gray-Weale, U. Bach, Y.-B. Cheng, L. Spiccia, Angew. Chem., Int. Ed. Engl. 2014, 53, 9898.

[30] N. J. Jeon, J. H. Noh, Y. C. Kim, W. S. Yang, S. Ryu, S. I. Seok, Nat. Mater. 2014, 13, 897.

[31] J.-W. Lee, H.-S. Kim, N.-G. Park, Acc. Chem. Res. 2016, 49, 311.

[32] N. Ahn, D.-Y. Son, I.-H. Jang, S. M. Kang, M. Choi, N.-G. Park, J. Am. Chem. Soc. 2015, 137, 8696.

[33] N. G. Park, Inorg. Chem. 2017, 56, 3.

[34] T. Bu, L. Wu, X. Liu, X. Yang, P. Zhou, X. Yu, T. Qin, J. Shi, S. Wang, S. Li, Z. Ku, Y. Peng, F. Huang, Q. Meng, Y.-B. Cheng, J. Zhong, Adv. Energy Mater. 2017, 7, 1700576.

[35] M. Zhang, Z. Wang, B. Zhou, X. Jia, Q. Ma, N. Yuan, X. Zheng, J. Ding, W.-H. Zhang, Sol. RRL 2018, 2, 1700213.

[36] X. Li, D. Bi, C. Yi, J.-D. Décoppet, J. Luo, S. M. Zakeeruddin, A. Hagfeldt, M. Grätzel, Science 2016, 353, 58.
[37] S. Sanchez, X. Hua, N. Phung, U. Steiner, A. Abate, Adv. Energy Mater. 2018, 8, 1702915.

[38] M. Saliba, T. Matsui, J.-Y. Seo, K. Domanski, J.-P. Correa-Baena, M. K. Nazeeruddin, S. M. Zakeeruddin, W. Tress, A. Abate, A. Hagfeldtd, M. Grätzel, Energy Environ. Sci. 2016, 9, 1989.

[39] B. Ding, Y. Li, S. Y. Huang, Q. Q. Chu, C. X. Li, C. J. Li, G. J. Yang, J. Mater. Chem. A 2017, 5, 6840.

[40] R. Munir, A. D. Sheikh, M. Abdelsamie, H. Hu, L. Yu, K. Zhao, T. Kim, O. E. Tall, R. Li, D.-M. Smilgies, A. Amassia, Adv. Mater. 2017, 29, 1604113

[41] B. Conings, A. Babayigit, M. T. Klug, S. Bai, N. Gauquelin, N. Sakai, J. T.-W. Wang, J. Verbeeck, H.-G. Boyen, H. J. Snaith, Adv. Mater. 2016, 28, 10701

[42] S. Song, M. T. Hörantner, K. Choi, H. J. Snaith, T. Park, J. Mater. Chem. A 2017, 5, 3812.

[43] M. Bouchard, J. Hilhorst, S. Pouget, F. Alam, M. Mendez, D. Djurado, D. Aldakov, T. Schülli, P. Reiss, J. Phys. Chem. C 2017, 121, 7596.

[44] Z. Li, M. Yang, J.-S. Park, S.-H. Wei, J. J. Berry, K. Zhu, Chem. Mater. 2016, 28, 284

[45] Y.-K. Ren, X.-H. Ding, Y.-H. Wu, J. Zhu, T. Hayat, A. Alsaedi, Y.-F. Xu, Z.-Q. Li, S.-F. Yang, S.-Y. Da, J. Mater. Chem. A 2017, 5, 20327.

[46] H. Yu, F. Wang, F. Xie, W. Li, J. Chen, N. Zhao, Adv. Funct. Mater. 2014, 24, 7102 .

[47] A. Z. Chen, B. J. Foley, J. H. Ma, M. R. Alpert, J. S. Niezgoda, J. J. Choi, J. Mater. Chem. A 2017, 5, 7796.

[48] S. De Wolf, J. Holovsky, S.-J. Moon, P. Löper, B. Niesen, M. Ledinsky, F.-J. Haug, J.-H. Yum, C. Ballif, J. Phys. Chem. Lett. 2014, 5, 1035.

[49] M. L. Theye, L. Chahed, P. R. I Cabarrocas, K. Zellama, Philos. Mag. B 1991, 63, 143

[50] L. Goris, A. Poruba, A. Purkrt, K. Vandewal, A. Swinnen, I. Haeldermans, K. Haenen, J. V. Manca, M. Vaněček, J. NonCryst. Solids 2006, 352, 1656.

[51] J. A. Carr, S. Chaudhary, Energy Environ. Sci. 2013, 6, 3414.

[52] L. K. J. Vandanne, R. Alabedra, M. Zonniti, Solid State Electron. $1983,26,671$.

[53] Z. Chobola, Microelectron. Reliab. 2001, 41, 1947.

[54] P. Calado, A. M. Telford, D. Bryant, X. Li, J. Nelson, B. C. O'Regan, P. R. F. Barnes, Nat. Commun. 2016, 7, 13831

[55] K. K. Wong, A. Fakharuddin, P. Ehrenreich, T. Deckert, M. Abdi-Jalebi, R. H. Friend, L. Schmidt-Mende, J. Phys. Chem. C 2018, 122, 10691.

[56] G. Yang, C. Wang, H. Lei, X. Zheng, P. Qin, L. Xiong, X. Zhao, Y. Yan, G. Fang, J. Mater. Chem. A 2017, 5, 1658

[57] M. F. Aygüler, A. G. Hufnagel, P. Rieder, M. Wussler, W. Jaegermann, T. Bein, V. Dyakonov, M. L. Petrus, A. Baumann, P. Docampo, ACS Appl. Mater. Interfaces 2018, 10, 11414.

[58] C. Wang, C. Xiao, Y. Yu, D. Zhao, R. A. Awni, C. R. Grice, K. Ghimire, I. Constantinou, W. Liao, A. J. Cimaroli, P. Liu, J. Chen, N. J. Podraza, C.-S. Jiang, M. M. Al-Jassim, X. Zhao, Y. Yan, Adv. Energy Mater. 2017, 7, 1700414

[59] S. D. Sung, D. P. Ojha, J. S. You, J. Lee, J. Kim, W. I. Lee, Nanoscale 2015, 7, 8898 .

[60] S. Shao, M. Abdu-Aguye, L. Qiu, L.-H. Lai, J. Liu, S. Adjokatse, F. Jahani, M. E. Kamminga, G. H. ten Brink, T. T. M. Palstra, B. J. Kooi, J. C. Hummelenab, M. A. Loi, Energy Environ. Sci. 2016, 9, 2444. 